\title{
Strates
}

STRATES Matériaux pour la recherche en sciences sociales

$11 \mid 2004$

Jeune recherche, la vitalité d'un laboratoire

\section{Les plateaux du sud-est du Bassin parisien, entre PAC 92 et Agenda 2000}

\section{Geneviève PIERRE}

\section{(2) OpenEdition \\ 1 Journals}

Édition électronique

URL : http://journals.openedition.org/strates/446

DOI : $10.4000 /$ strates. 446

ISSN : $1777-5442$

Éditeur

Laboratoire Ladyss

Édition imprimée

Date de publication : 1 janvier 2004

ISSN : 0768-8067

\section{Référence électronique}

Geneviève PIERRE, "Les plateaux du sud-est du Bassin parisien, entre PAC 92 et Agenda 2000 », Strates [En ligne], 11 | 2004, mis en ligne le 14 janvier 2005, consulté le 08 septembre 2020. URL http://journals.openedition.org/strates/446 ; DOI : https://doi.org/10.4000/strates.446

Ce document a été généré automatiquement le 8 septembre 2020

Tous droits réservés 


\title{
Les plateaux du sud-est du Bassin parisien, entre PAC 92 et Agenda 2000
}

\author{
Geneviève PIERRE
}

1 Thèse de géographie soutenue à l'université de Paris X-Nanterre en juin 2001 sous la direction de Jean-Paul CHARVET, professeur des universités (574 p.)

2 La PAC 92 constitue un tournant majeur dans la définition des mécanismes agricoles européens, en mettant au centre les paiements compensatoires à la surface cultivée en COP (Céréales et oléo-protéagineux), le gel obligatoire des terres et les mesures agrienvironnementales. Elle touche principalement les espaces agricoles de grandes cultures (céréales et oléagineuses), notamment le Bassin parisien. Or, toutes les régions agricoles du Bassin parisien ne sont pas touchées de la même manière.

3 Les plateaux du sud-est du Bassin parisien (plateaux de Bourgogne, de Langres et du Barrois de la Haute-Marne) représentent un cas extrême d'adaptation à la PAC 92 et à ses prolongements actuels. Les choix culturaux et productifs y sont quasi exclusivement orientés vers les productions soumises à la réforme de la PAC 92 (les COP) ou étroitement contrôlées par la PAC : élevage bovin lait et viande. Dans ces «petites terres » aux sols superficiels, à faible réserve hydrique et contenant une forte proportion de cailloux, le potentiel de rendement est bien plus faible qu'au centre du Bassin parisien, pour des possibilités de diversification beaucoup plus réduites: impossibilité de pratiquer les betteraves industrielles, les pommes de terres, les pois protéagineux ou le maïs-grain. Ces contraintes doivent être repensées en fonction de la concurrence accrue entre les différents espaces de grandes cultures exportateurs de matières premières agricoles, face à la diminution des prix garantis et à la mondialisation des marchés agricoles. Cependant, par la PAC 92, la valeur agricole du sol cultivable prend une autre signification : elle dépend aussi des droits à produire et à primes qui y sont attachés. Aussi les petites terres prennent une valeur économique et administrative inespérée, sans que la "valeur agronomique du sol» ait subi de changement. 
4 Parallèlement, la nature même du système de production et du modèle agricole de développement mis en place depuis les années cinquante, par l'agrandissement continu des superficies d'exploitation, en grandes cultures et système mixte, limite les possibilités de diversification. L'agrandissement des exploitations a permis une remise en valeur complète, voire un élargissement, par défrichement, de l'ensemble de cet espace agricole de production, livré à la friche au début des années cinquante, après des décennies de crise et de déshérence. Cet effort est symbolisé par l'activité de la SAFE (Société d'aménagement des friches de l'est créée en 1958 avec l'impulsion d'E. Pisani sous le nom de SEFE - Société d'étude des friches et taillis pauvres de l'est) dans 13 départements de l'Est de la France, dont l'Yonne, la Côte d'Or et la Haute-Marne, dès les années soixante. Le vide démographique, la désertification de l'espace agricole ont été la cause première (et non la conséquence première) de l'agrandissement des exploitations. Cette idée a été évoquée, dès les années quatre-vingt, par Violette Rey (1980) au sujet du Berry. La perpétuation du modèle, à partir des années soixante-dixquatre-vingt, contribue à accentuer un système dans lequel la volonté de repousser les limites de la productivité par actif agricole crée, par la très faible densité d'actifs agricoles, une rigidité importante dans l'évolution du système de production et entrave la diversification.On doit reconnaître des nuances internes dans les plateaux du sud-est $\mathrm{du}$ Bassin parisien. Les régions et départements incluant l'auréole des plateaux de «terres à cailloux » (des terres de groies du Poitou-Charentes jusqu'à la Lorraine) se sont reconnus dans l'appartenance "revendiquée " à une zone intermédiaire, en France, par rapport à leur système de production agricole et ses potentialités (rendements) en grandes cultures.Les plateaux «à cailloux" s'inscrivent entre la France des grandes cultures et la France de l'élevage. Les plateaux participent euxmêmes de cette transition puisque, de la Champagne du Berry aux plateaux de Langres et $\mathrm{du}$ Barrois, les exploitations en système mixte et pratiquant l'élevage bovin apparaissent de plus en plus représentées. C'est surtout la question du calcul des rendements de référence, au début des années quatre-vingt-dix, fondamentale pour la définition des primes PAC et du revenu agricole, qui a inquiété ces départements, chacun adoptant cependant un rendement unique : Yonne, Côte d'Or, Cher, Indre, départements du Poitou-Charente ou de Lorraine. Cependant, la définition des rendements de référence a été plus défavorable aux départements du centre du Bassin parisien qu'à ceux des marges intermédiaires du sud et de l'est. Dans l'Yonne, ce système à rendement unique départemental a été favorable aux plateaux de Bourgogne, par comparaison avec les régions de grandes cultures du Nord du département.

5 Le caractère de "zone intermédiaire » a été également défini par rapport à la faible métropolisation, au vide démographique, à la faible polarisation de l'espace (Bavoux, 1993, 1994) dans les marges sud et est du Bassin parisien : un espace flou, une zone d'indécision entre différentes influences urbaines, celles de la région parisienne, de la région lyonnaise et du système multipolarisé de la France du nord-est. Les plateaux de Bourgogne, de Langres et du Barrois constituent plus généralement un angle mort des zones intermédiaires du sud-est du Bassin parisien, malgré des nuances locales liées à la périurbanisation ou l'influence urbaine à partir de Dijon, Auxerre, voire Chaumont.

6 Ce caractère intermédiaire en France peut être également attesté par l'articulation développement rural - développement agricole, montré par les travaux de la SEGESA au début des années quatre-vingt-dix (Bontron, Cabanis, Velard, 1992; Bontron, Cabanis, 1993). Les cantons ruraux fragiles des plateaux ne présentent pas les cas les 
plus graves par rapport à d'autres exemples où la fragilité est plus générale (le Morvan). Sur les plateaux, l'agriculture est jugée capable économiquement de se pérenniser ou se moderniser rapidement.

7 L'articulation entre développement agricole et développement rural local interroge profondément les espaces de grandes cultures du sud-est du Bassin parisien, au moment où les politiques agricoles, tant en France qu'en Europe, s'orientent dans une réflexion autour de la notion de politiques intégrées, au service de la durabilité de l'activité économique et des territoires : LOA (Loi d'orientation agricole) de 1999 (avec les CTE ou Contrats territoriaux d'exploitation), base de la multifonctionnalité de l'agriculture, la loi d'aménagement du territoire de 1999 et la définition des pays, l'Agenda 2000 dans l'UE, l'accord de Berlin et la réforme des fonds structurels intégrant développement rural et agricole dans un même ensemble de mesures, au lieu de les juxtaposer. A priori, le modèle de développement agricole du sud-est du Bassin parisien, particulièrement sur les plateaux de Bourgogne, est à l'opposé de ces définitions nouvelles. Les plateaux ont développé une logique de développement agroexportatrice, productrice de matières premières locales, à très faible valeur ajoutée locale.

8 Certes, des revenus garantis, payés à la surface, dans un système où la SAU (Surface agricole utile) par actif est élevée, ont permis une évolution très favorable des revenus notamment dans les premières années de la réforme où le cours des productions (COP) a été plus élevé que prévu : l'agrandissement accéléré des exploitations et de la SAU par actif agricole a fortement contribué à la définition du revenu. La nature même des aides PAC encourage la tendance lourde du développement agricole des plateaux à la consommation de foncier dans des systèmes pratiquant la simplification à outrance : simplification des paysages, hyper spécialisation en blé, orge, colza, simplification des pratiques culturales.

9 Cela révèle surtout une dépendance extérieure accrue dans la constitution du revenu, puisque dans certains cas, les paiements PAC représentent plus de $90 \%$ de l'EBE (l'Excédent brut d'exploitation égal à : VA + subventions d'exploitation + produits divers - charges de structure). La dépendance est de deux ordres : politique, puisque suspendue aux décisions budgétaires de Bruxelles, et économique, par la mondialisation des cours des productions agricoles.

10 Parallèlement, la nature des aides directes au revenu, payées par le contribuable et non plus principalement par le consommateur comme c'était le cas avant 1992, introduit une exigence de transparence et une demande sociale d'environnement, de production de qualité.

11 Or, si c'est bien la PAC 92 qui introduit ce changement culturel fort dans les politiques agricoles, les premières années de la réforme ont contribué à sous-estimer la dépendance extérieure. Celle-ci apparaît nettement lors de la redéfinition des politiques agricoles et de ses financements par l'accord de Berlin, dans le cadre de l'Agenda 2000, et par la LOA de 1999. Cela affecte beaucoup les plateaux du sud-est du Bassin parisien : la modulation des aides directes les touche en priorité, ainsi que la place du colza dont les primes compensatrices vont être ramenées à celles des céréales, dans un contexte de baisse des cours mondiaux... Comment l'orientation du système agricole de production depuis la PAC 92 peut-elle s'avérer compatible avec les exigences croissantes de multifonctionnalité, de durabilité, de production de qualité, 
territorialisée, de traçabilité alors que les logiques de développement agricole et rural apparaissent très souvent décalées voire antinomiques ?

Surtout, quel type de durabilité définir, à quelle échelle? Peut-on améliorer le productivisme, par l'écoconditionnalité, pour le rendre compatible avec l'agriculture durable (Briel, Vilain, 1999)?

Le bilan de la PAC 92 dans les plateaux du sud-est du Bassin parisien révèle, parfois de façon très accusée, des infléchissements dans les pratiques agricoles en grandes cultures. Surtout, certains aspects de la PAC 92 ont reçu un écho particulièrement fort dans la zone d'étude. Ainsi, la tendance lourde à l'agrandissement a-t-elle été confortée, au détriment de l'installation, grâce aux compensations par hectare de COP mais également à la première mesure de préretraite agricole (1992-95) pour les exploitants de 55 à 59 ans (mesure sociale d'accompagnement à la PAC 92). Toutefois, l'impact de cette mesure doit être relativisé en fonction de la structure par âge des exploitants (souvent âgés à la veille de la réforme).

Si les systèmes de production de la zone d'étude apparaissent moins intensifs, en grandes cultures, que ceux du centre du Bassin parisien, on ne peut guère parler d'extensification ou de diffusion d'un modèle nettement extensif. Les pratiques économes en matière de gestion des coûts de production, le discours et les pratiques de l'agriculture raisonnée s'y sont bien implantés, souvent même avant la PAC 92, par le travail parallèle des chambres d'agriculture et des organismes stockeurs. L'application de la directive nitrates dans les années quatre-vingt-dix, concernant les zones vulnérables (l'essentiel des plateaux calcaires du sud-est du Bassin parisien), demande peu d'efforts particuliers aux agriculteurs habitués à utiliser des doses d'engrais en dessous ou à la limite des préconisations. Pourtant, l'impact des MAE (les Mesures agrienvironnementales) a été faible.

15 Le principal effet de la PAC 92 et des accords du GATT, c'est, face à la volatilité des cours et la baisse des prix garantis, la volonté, pour l'agriculteur, de prendre davantage en mains les conditions de commercialisation de sa production et sécuriser le plus possible son revenu par une politique de qualité et de traçabilité impulsée par les organismes stockeurs. Sur les plateaux, la véritable révolution n'est pas dans la diversification - difficile ici - mais dans les conditions mêmes de production de masse des quelques matières premières agricoles pratiquées (blé, orge et colza) : une production de plus en plus réalisée sous contrat de qualité, pour des variétés spécifiques, avec un taux de protéine généralement élevé en blé tendre, répondant aux exigences de certains marchés. Les clients réclament de plus en plus une qualité immédiatement identifiable, homogène et garantie, issue d'un processus de production certifié. La production sous contrat représente d'ores et déjà plus de $40 \%$ de la quantité de blé réceptionnée chez certains organismes stockeurs des plateaux. Cependant, une politique de développement agricole intégrée à l'ensemble du développement local a du mal à s'imposer, alors même que, sans l'agriculture, il n'y a souvent plus de durabilité rurale envisageable.

Pourtant, il est nécessaire de définir, entre les différentes régions agricoles des plateaux, des nuances dans les dépendances à la PAC, dans l'hyper spécialisation en grandes cultures, dans les stratégies d'agrandissement (à l'intérieur de formes sociétaires collectives ou non), dans l'articulation entre le développement territorial rural et la pérennité de l'activité économique agricole. 
Pour schématiser, on peut distinguer deux systèmes, sur les plateaux : les plateaux de Bourgogne de l'Yonne et le plateau de Langres « central », particulièrement la " grande Montagne" haut-marnaise (cantons d'Auberive surtout, voire Prauthoy). Dans le premier cas, le décalage entre les logiques de développement agricole et rural apparait souvent très net alors que l'on se trouve dans le milieu rural le moins fragile de l'ensemble de l'espace d'étude. La croissance démographique récente (1990-1999) a été plutôt positive, en lien avec la diffusion urbaine et périurbaine à partir d'Auxerre et la forte proportion de retraités dans la population. Le développement agricole repose, plus qu'ailleurs, sur le modèle agro-exportateur, pratiquant l'hyper spécialisation et la simplification à l'extrême des paysages, malgré un contre-exemple important: de Coulanges la Vineuse à Chablis et Tonnerre, le développement de la viticulture de qualité et des paysages labellisés des cerisiers de la vallée de l'Yonne peuvent créer un point d'appui, très localisé, au développement rural par le tourisme. Le cas du Tonnerrois permet de faire le lien entre le système de production agricole très dominant des plateaux et l'existence de productions territorialisées - dont les revenus sont indépendants de la PAC -, puisque les viticulteurs actuels sont également en grande partie des céréaliers. Ici, la dynamique actuelle du développement rural place l'activité viticole au cœur de ses projets, en espérant créer une économie touristique modeste.

En "grande Montagne » haut-marnaise, la fragilité rurale apparaît la plus grande de l'espace d'étude envisagé, alors que le système de production agricole dispose des revenus par hectare de SAU les plus faibles. Or, des projets d'agriculture multifonctionnelle et territorialisée tentent de se mettre en place et la volonté de raisonner au niveau du territoire, bien que balbutiante, apparaît mieux représentée qu'ailleurs dans ces plateaux : un PDD (plan de développement durable) a vu le jour dans les années 90, la définition de deux $\mathrm{AOC}$ fromagères sur les cantons d'Auberive et de Prauthoy (Epoisses et Langres) permet d'envisager des projets de CTE (Contrats territorials d'exploitation) collectifs et de «territorialiser " une partie du revenu ; une démarche de pays est en train de se concrétiser.

Ces deux exemples peuvent illustrer les possibilités d'évolution, dans un avenir proche, des différents espaces agricoles dans leur environnement rural. Si l'agrandissement accéléré a pu inquiéter, en aucun cas on ne peut en déduire un risque de déprise. Les solutions juridiques sociétaires, accélérées dans les années quatre-vingt-dix (surtout les EARL), peuvent constituer une solution à l'utilisation de l'espace agricole. Toutefois, pour qu'une réelle extensification se développe, il faudrait un agrandissement encore plus rapide et des libérations de terres plus importantes (pour un impact social qui inquiète) alors qu'aujourd'hui, on se trouve en situation de blocage foncier. Si le maintien du potentiel de production semble acquis, le décalage entre le développement agricole et rural peut s'accentuer, voire les rendre antinomiques. 


\section{BIBLIOGRAPHIE}

Bavoux (J. J.), « La Bourgogne, un archétype d'espace intermédiaire? » Annales de géographie no 570, 1993, p. 162-174.

INDEX

Keywords : France

Mots-clés : Agriculture 